\title{
Extended Imaging Protocols to Elucidate Sources of Cardiovascular Embolism in the Work-up of Ischemic Stroke
}

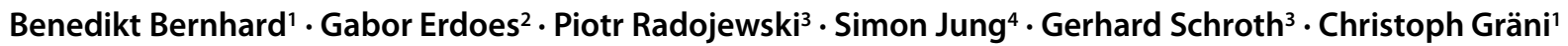

Accepted: 14 September 2021

๑) Springer-Verlag GmbH Germany, part of Springer Nature 2021

\section{Editorial}

Ischemic stroke is an important cause of morbidity and mortality increasingly contributing to disability-adjusted lifeyears worldwide [1]. According to the Trial of Org 10172 in Acute Stroke Treatment (TOAST) classification [2], the etiology of ischemic stroke includes atherosclerosis of the large arteries, small-vessel occlusion, cardioembolism and stroke of other determined etiology (e.g. related to cardiovascular interventions) and cryptogenic stroke. Cryptogenic stroke comprises up to about $25 \%$ of ischemic stroke and is presumed to be mostly of embolic origin (about $17 \%$ of all ischemic stroke [3]). This leads to the definition of embolic stroke of undetermined source (ESUS), which is characterized by non-lacunar location in the absence of proximal arterial stenosis or an identified source of cardiac embolism [4]. While other stroke entities have plain therapeutic targets, ESUS incorporates various underlying diseases and entities for which medical, surgical or interventional treatment might be available but cannot be applied due to the lack of delineation. Several attempts were made to address this issue; however, despite recent improvements in imaging modalities and the diagnostic work-up, ESUS remains a relevant concern [3]. Presumed origins of ESUS are mostly located in the heart, such as in the left atrial

Christoph Gräni

christoph.graeni@insel.ch

1 Department of Cardiology, Inselspital, Bern University Hospital, University of Bern, 3010 Bern, Switzerland

2 Department of Anaesthesiology and Pain Medicine, Inselspital, Bern University Hospital, University of Bern, Bern, Switzerland

3 Institute of Diagnostic and Interventional Neuroradiology, Inselspital, Bern University Hospital, University of Bern, Bern, Switzerland

4 Department of Neurology, Inselspital, University Hospital and University of Bern, Bern, Switzerland appendage (LAA), particularly in the setting of atrial fibrillation, in the left ventricle (LV), particularly in the setting of ischemic heart disease and concomitant impaired cardiac function with local blood stasis, on left ventricular native valves/valve prostheses or are from neoplasms (for other common sources of cardiac embolism please refer to Fig. 1; $[5,6])$. Deep vein thrombosis in connection with right-to-left shunting and thus embolism through a patent foramen ovale (PFO) or atrial septal defect (ASD), is another important entity. Apart from cardiac sources, aortic arch atheroma and aortic arch aneurysm or tumors other than primary or secondary cardiac neoplasm may also be sources of embolism that can cause ischemic stroke and can be targeted by specific treatment.

The primary goal of imaging in the acute setting of stroke is to rule out intracerebral and/or intracranial hemorrhages, usually by native computed tomography (CT) or magnetic resonance (MR) imaging, in order to initiate timely treatment $[5,6]$. Imaging of the vessels supplying the brain by means of CT or MR angiography to determine the location of the vessel occlusion and optimize the access routes and techniques of endovascular thrombectomy is another integral part of acute stroke work-up. Additional techniques of advanced neuroimaging, such as perfusion CT/MR imaging, are mainly to be used for unclear or complex situations, such as wake-up stroke, stroke of unknown onset, or stroke of the posterior circulation and coma [7].

However, none of these modalities provide the possibility to systematically assess extracerebral sources of ischemic stroke and therefore should be followed by specified cardiac imaging in the following days in order to optimize secondary prevention [8]. Echocardiography, especially transthoracic echocardiography (TTE), can identify many sources of cardiac embolism and appears reasonable as a first line cardiac imaging modality in most patients. It can be useful to supplement the TTE with transesophageal echocardiography (TEE) image acquisition, as TEE offers a better visualization of certain cardiac structures, especially if aortic pathology, prosthetic valve dys- 
Fig. 1 Common sources of cardiac embolism causing ischemic stroke (modified from freely available Servier Medical Art templates, smart.servier.com)

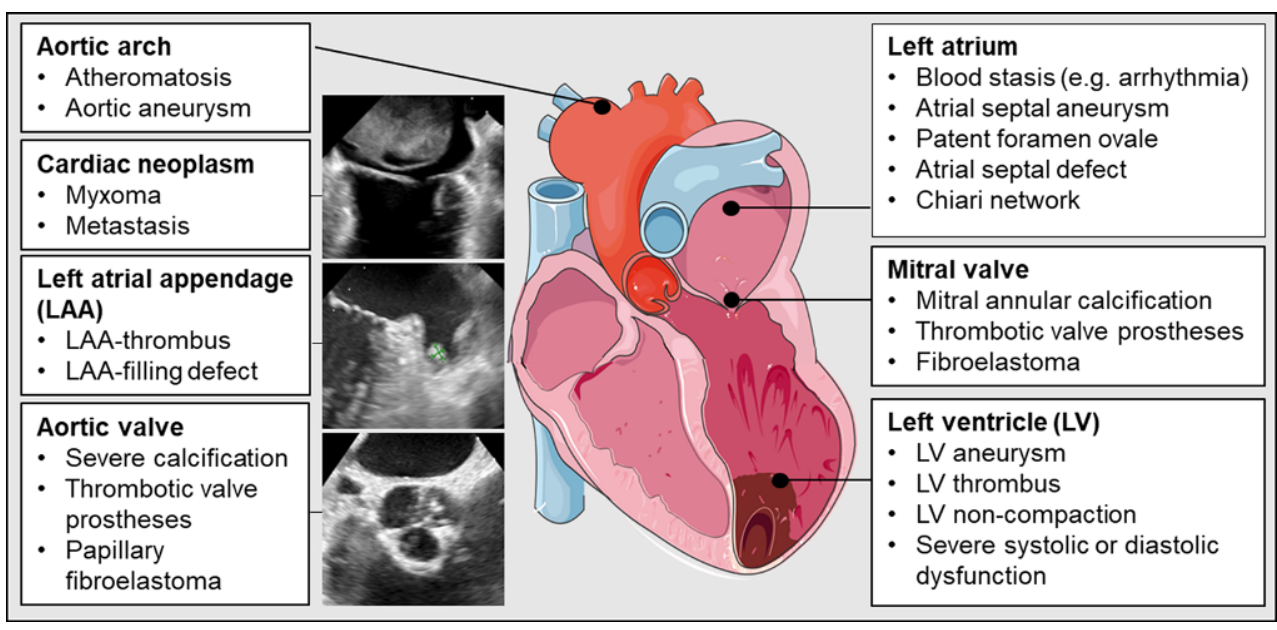

function, valvular endocarditis, atrial septal defect, PFO or a LAA thrombus is suspected. However, echocardiography is usually performed after treatment of acute stroke, resulting in a relevant time delay from clinical presentation to the identification of potential sources of cardiac embolism by echocardiography.

Addressing these concerns, we congratulate the two studies presented by Molina-Fuentes et al. [9] and Austein et al. [10] aiming to develop novel extended CT protocols to capture cardiovascular sources in the acute phase of ischemic strokes. The authors aimed at a further delineation of ESUS by incorporating a cardiac CT (CCT) additional to standard brain $\mathrm{CT}$ acquisition in patients with acute ischemic stroke presenting at the emergency department. Together 169 patients were included in their studies of whom 109 underwent concomitant echocardiography after a median of 3 (Austein et al.) and 9 days (MolinaFuentes et al.). Among these 109 patients, CCT identified 22 sources of embolism ( $n=9$ LAA thrombi, $n=5$ valve thrombi, $n=3$ thrombi in the aortic arch, $n=3 \mathrm{LV}$ thrombi, $n=1 \mathrm{ASD}$, and $n=1$ myxoma). Echocardiography missed more than $50 \%$ of sources of embolism that were found in the CCT (4/10 and 5/12, respectively), while the specificity of echocardiography was $100 \%$. The additional radiation exposure with the new protocols was on an acceptable level ( +265 and $+33.6 \mathrm{mGy}^{*} \mathrm{~cm}$, respectively). Most important limitation of both retrospective studies was the lack of a systematic echocardiography assessment (i.e. in the studies the authors used a mix of TTE and TEE) and the lack of a control group that could have allowed a comparison of standard care to the new protocols. The findings missed in echocardiography can be related to lower diagnostic accuracy of TTE compared to CCT or be related to the time-delay between CCT and echocardiography. Intracardiac thrombi might have dissolved or embolized after administration of thrombolytic therapy before echocardiographic image acquisition took place. Despite these limi- tations, both studies were able to prove their concepts and generated the hypothesis that these new protocols could help to reduce the number of ESUS by identifying sources of cardiac embolism with potential clinical impact. ESUS patients are at a considerable $4.5 \%$ risk of having another stroke within the following 3 years [3], underlining the need for further tailoring of medical treatment. The treatment of unselected ESUS patients with direct oral anticoagulants (DOAC), however, is associated with an extensive increase in major bleeding events (hazard ratio, HR 2.72; $95 \%$ confidence interval, CI 1.68-4.39) [11], without a relevant reduction in stroke recurrence $[11,12]$. Hence, ESUS patients are mostly treated with antiplatelet therapy only [3]. Identification of cardiac sources of embolism (e.g. LV or LAA thrombus) by new protocols can have relevant therapeutic implications and might justify an escalation of medical treatment from antiplatelets to DOACs in selected patients. We therefore see the value of the new protocols particularly in the identification of such intracardiac thrombi that might be missed by echocardiography but would require anticoagulant treatment in a timely manner. Structural sources of cardiac embolism (e.g., cardiac tumors, aortic valve calcification, PFO) on the other hand, can reliably be diagnosed by delayed echocardiography and treated after neurologic recovery. Further strengths of the new protocols are their noninvasive character with a fast and ease image acquisition without the need for deep sedation or even general anesthesia. This might save costs, reduce complications and lead to earlier detection of sources of cardiac embolism.

It remains a matter of debate, which sequences an additional CCT should be incorporate in the setting of acute stroke patients. Retrospective electrocardiogram (ECG) gating, as applied in both studies, comes at the expense of higher radiation exposure but may provide best image quality with minimizing artefacts $[13,14]$. Prospective ECGgated image acquisition has the advantage of limiting additional radiation dose with good image quality. Non-gated 
CCT, as suggested by other authors [15], might be useful too, as outlined by a previous trial applying non-gated CT in a similar setting [16]. Furthermore, the addition of a delayed phase acquisition (as applied in both studies) might improve differentiation between thrombus and flow artefact. In fact, compared to TEE as reference standard, diagnostic accuracy for LAA detection improves from $94 \%$ to $99 \%$ in delayed imaging due to higher specificity [17]. It can be hypothesized that delayed CCT can even be more accurate than TEE in the detection of LAA thrombi, since previous studies excluded patients with poor image quality in TEE, and the lack of another reference standard comparing both modalities.

Apart from CCT, MR imaging can also be applied in the work-up of stroke and help to identify the sources of embolism and provide diagnostic advantages in determining stroke etiology. We congratulate Spuentrup et al. who presented a case series and demonstrate the use of a molecular imaging with a fibrin-specific MR imaging contrast agent in the identification of the source of thromboembolic stroke in 4 patients [18]. Fibrin-binding contrast agent might not only improve thrombus visualization but can also identify plaque and plaque rupture as cause for ischemic stroke. Previous work could demonstrate the feasibility of this approach in 1.5 T MR imaging [19], while the current case series describes its use in 3.0 T MR imaging. Incorporating the application of such molecular contrast agents into MR protocols for acute stroke into routine clinical practice is so far limited by their high costs and the lack of larger prospective randomized-controlled trials demonstrating a superiority over standard image acquisition. Furthermore, MR imaging is a time-intense and cost-intense modality with several contraindication, such as severe orthopnea, recent device implantation and claustrophobia.

It has to be taken into account that not all causes of cardiac embolism as potential cause for ESUS are detectable by an additional cardiac CT or by contrast MR imaging. Paroxysmal atrial fibrillation with embolized LAA thrombus, smaller atrial shunts, and atrial blood stasis are entities that require other diagnostic approaches, such as echocardiography or prolonged rhythm monitoring. Neither CCT nor contrast-enhanced MR imaging can replace these modalities if the initial imaging remains without persuasive findings in patients with ESUS. Furthermore, it must be taken into account that these new protocols require established interdisciplinary expertise between neurology, radiology, neuroradiology, and cardiology to optimize the imaging in the emergency setting. Before introduction into clinical practice, the proposed approaches require validation in prospective randomized trials. So far, the mentioned studies did confirm feasibility of the new techniques in selected patients but do not allow stronger conclusions due to the lack of a control group and the exclusion of patients with other potential causes for stroke, such as atrial fibrillation without anticoagulant treatment, hemorrhagic stroke, periprocedural stroke, carotid atheromatosis or hypercoagulatory disease. It will be of great interest to investigate whether the new protocols can reduce the number of ESUS (by identifying sources of cardiac embolism) in the stroke population, and if they are associated with better outcome due to optimized medical treatment, or if they might expose the patient to unnecessary additional radiation and delay stroke therapy.

Conflict of interest B. Bernhard, G. Erdoes, P. Radojewski and S. Jung declare that they have no competing interests. G. Schroth is member of DSMB for PROOF and CEC member for Medtronic Neurovascular Inspire, Stryker IMPACT and Ceronovus perfect studies. C. Gräni receives funding from the Swiss National Science Foundation (SNF) and InnoSuisse, outside of the submitted work.

\section{References}

1. Krishnamurthi RV, Moran AE, Feigin VL, Barker-Collo S, Norrving B, Mensah GA, Taylor S, Naghavi M, Forouzanfar MH, Nguyen G, Johnson CO, Vos T, Murray CJ, Roth GA; GBD 2013 Stroke Panel Experts Group. Stroke Prevalence, Mortality and DisabilityAdjusted Life Years in Adults Aged 20-64 Years in 1990-2013: Data from the Global Burden of Disease 2013 Study. Neuroepidemiology. 2015;45:190-202. Erratum in: Neuroepidemiology. 2016;46:181.

2. Adams HP Jr, Bendixen BH, Kappelle LJ, Biller J, Love BB, Gordon DL, Marsh EE 3rd. Classification of subtype of acute ischemic stroke. Definitions for use in a multicenter clinical trial. TOAST. Trial of Org 10172 in Acute Stroke Treatment. Stroke. 1993 Jan;24:35-41.

3. Hart RG, Catanese L, Perera KS, Ntaios G, Connolly SJ. Embolic Stroke of Undetermined Source: A Systematic Review and Clinical Update. Stroke. 2017;48:867-72.

4. Hart RG, Diener HC, Coutts SB, Easton JD, Granger CB, O'Donnell MJ, Sacco RL, Connolly SJ; Cryptogenic Stroke/ESUS International Working Group. Embolic strokes of undetermined source: the case for a new clinical construct. Lancet Neurol. 2014;13:42938 .

5. Ntaios G, Perlepe K, Lambrou D, Sirimarco G, Strambo D, Eskandari A, Karagkiozi E, Vemmou A, Koroboki E, Manios E, Makaritsis K, Vemmos K, Michel P. Prevalence and Overlap of Potential Embolic Sources in Patients With Embolic Stroke of Undetermined Source. J Am Heart Assoc. 2019;8:e12858.

6. Ntaios G, Papavasileiou V, Milionis H, Makaritsis K, Manios E, Spengos K, Michel P, Vemmos K. Embolic strokes of undetermined source in the Athens stroke registry: a descriptive analysis. Stroke. 2015;46:176-81.

7. Turc G, Bhogal P, Fischer U, Khatri P, Lobotesis K, Mazighi M, Schellinger PD, Toni D, de Vries J, White P, Fiehler J. European Stroke Organisation (ESO) - European Society for Minimally Invasive Neurological Therapy (ESMINT) Guidelines on Mechanical Thrombectomy in Acute Ischaemic StrokeEndorsed by Stroke Alliance for Europe (SAFE). Eur Stroke J. 2019;4:6-12.

8. Powers WJ, Rabinstein AA, Ackerson T, Adeoye OM, Bambakidis NC, Becker K, Biller J, Brown M, Demaerschalk BM, Hoh B, Jauch EC, Kidwell CS, Leslie-Mazwi TM, Ovbiagele B, Scott PA, Sheth KN, Southerland AM, Summers DV, Tirschwell DL. Guidelines for the Early Management of Patients With Acute Ischemic Stroke: 2019 Update to the 2018 Guidelines for the Early Management of 
Acute Ischemic Stroke: A Guideline for Healthcare Professionals From the American Heart Association/American Stroke Association. Stroke. 2019;50:e344-418. Erratum in: Stroke. 2019;50:e4401.

9. Molina-Fuentes MF, Neumann R, Behringer W, Franz M, Schulze PC, Witte OW, Günther A, Klingner C, Lehmkuhl L, Steiniger B, Teichgräber U, Rod JE, Mayer TE. Feasibility of the Big 5-Jena eCS Protocol : First Experience Implementing a New Extended CT Protocol in the Initial Diagnostics of Ischemic Stroke. Clin Neuroradiol. 2021; https://doi.org/10.1007/s00062-021-01058-6.

10. Austein F, Eden M, Engel J, Lebenatus A, Larsen N, Both M, Piesch TC, Salehi Ravesh M, Meyne J, Jansen O, Langguth P. Practicability and Diagnostic Yield of One-Stop Stroke CT with DelayedPhase Cardiac CT in Detecting Major Cardioembolic Sources of Acute Ischemic Stroke : A Proof of Concept Study. Clin Neuroradiol. 2021; https://doi.org/10.1007/s00062-021-01003-7.

11. Hart RG, Sharma M, Mundl H, Kasner SE, Bangdiwala SI, Berkowitz SD, Swaminathan B, Lavados P, Wang Y, Wang Y, Davalos A, Shamalov N, Mikulik R, Cunha L, Lindgren A, Arauz A, Lang W, Czlonkowska A, Eckstein J, Gagliardi RJ, Amarenco P, Ameriso SF, Tatlisumak T, Veltkamp R, Hankey GJ, Toni D, Bereczki D, Uchiyama S, Ntaios G, Yoon BW, Brouns R, Endres M, Muir KW, Bornstein N, Ozturk S, O'Donnell MJ, De Vries Basson MM, Pare G, Pater C, Kirsch B, Sheridan P, Peters G, Weitz JI, Peacock WF, Shoamanesh A, Benavente OR, Joyner C, Themeles E, Connolly SJ; NAVIGATE ESUS Investigators. Rivaroxaban for Stroke Prevention after Embolic Stroke of Undetermined Source. N Engl J Med. 2018;378:2191-201.

12. Diener HC, Sacco RL, Easton JD, Granger CB, Bernstein RA, Uchiyama S, Kreuzer J, Cronin L, Cotton D, Grauer C, Brueckmann M, Chernyatina M, Donnan G, Ferro JM, Grond M, Kallmünzer B, Krupinski J, Lee BC, Lemmens R, Masjuan J, Odinak M, Saver JL, Schellinger PD, Toni D, Toyoda K; RE-SPECT ESUS Steering Committee and Investigators. Dabigatran for Prevention of Stroke after Embolic Stroke of Undetermined Source. N Engl J Med. 2019;380:1906-17.

13. Wagner M, Butler C, Rief M, Beling M, Durmus T, Huppertz A, Voigt A, Baumann G, Hamm B, Lembcke A, Vogtmann T. Comparison of non-gated vs. electrocardiogram-gated 64-detectorrow computed tomography for integrated electroanatomic mapping in patients undergoing pulmonary vein isolation. Europace. 2010;12:1090-7.

14. Desjardins B, Kazerooni EA. ECG-gated cardiac CT. AJR Am J Roentgenol. 2004;182:993-1010.

15. Yeo LLL, Sia CH, Leow AST, Tan BYQ. Letter to the editor: Practicability and diagnostic yield of one-stop stroke CT with delayedphase cardiac CT in detecting major cardioembolic sources of acute ischemic stroke. Clin Neuroradiol. 2021; https://doi.org/10.1007/ s00062-021-01027-z.

16. Yeo LLL, Holmin S, Andersson T, Lundström E, Gopinathan A, Lim EL, Leong BSH, Kuan WS, Ting E, Tan BYQ, Eide SE, Tay ELK. Nongated Cardiac Computed Tomographic Angiograms for Detection of Embolic Sources in Acute Ischemic Stroke. Stroke. 2017;48:1256-61.

17. Romero J, Husain SA, Kelesidis I, Sanz J, Medina HM, Garcia MJ. Detection of left atrial appendage thrombus by cardiac computed tomography in patients with atrial fibrillation: a meta-analysis. Circ Cardiovasc Imaging. 2013;6:185-94.

18. Spuentrup E, Botnar R, Binder A, Katoh M, Spüntrup C. Molecular MR-Imaging in Thromboembolic Stroke Using a Fibrin-Specific Contrast Agent in Patients at 3 Tesla. Clin Neuroradiol. 2021; https://doi.org/10.1007/s00062-021-01052-y.

19. Vymazal J, Spuentrup E, Cardenas-Molina G, Wiethoff AJ, Hartmann MG, Caravan P, Parsons EC Jr. Thrombus imaging with fibrin-specific gadolinium-based MR contrast agent EP-2104R: results of a phase II clinical study of feasibility. Invest Radiol. 2009;44:697-704. 